\title{
„Ich bin wirklich glücklich zu preisen, einen solchen Verleger-Freund zu besitzen“" \\ Aspekte mathematischen Publizierens im Kaiserreich und in der Weimarer Republik
}

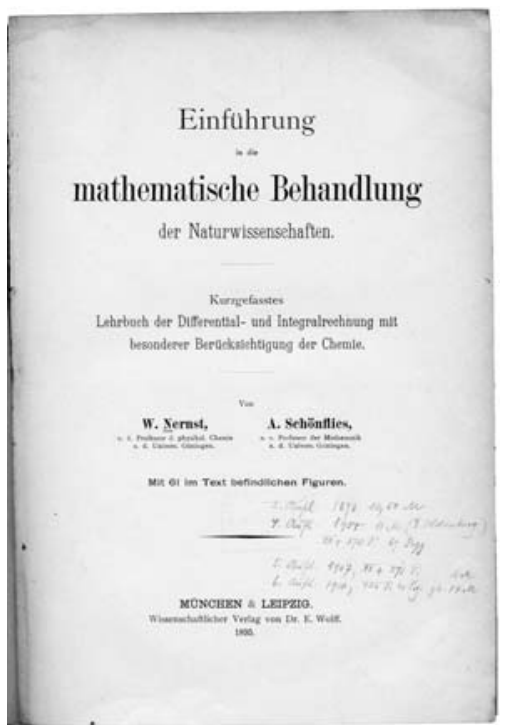

Titelseite von Walther Nernst und Arthur Schoenflies: Einführung in die mathematische Behandlung der Naturwissenschaften, 1895

\section{von Volker R. Remmert und Ute Schneider}

Dieser Beitrag ist die überarbeitete Fassung eines Vortrags von Volker Remmert am Mathematischen Institut der Universität Mainz im Juli 2006. Er ist aus unserem gemeinsamen DFG-Projekt Eine Disziplin und ihre Verleger: Formen, Funktionen und Initiatoren mathematischen Publizierens in Deutschland, 1871-1949 hervorgegangen.

1889 schrieb der Gründer der Zeitschrift für Mathematik und Physik Oskar Schlömilch (1823-1901) an den Vieweg Verlag in Braunschweig:

Daß Geodäten, Forstleute, Architekten, Ingenieure, Astronomen, Physiker etc. nicht wenig Höhere Mathematik brauchen, ist bekannt $u$. aus den betreffenden Fachschriften ersichtlich; selbst die Chymiker, die sich früher bei dem Worte Mathematik schreckensbleich bekreuzigten, fangen schon ganz hübsch an, zu differenzieren $\mathrm{u}$. $\mathrm{zu}$ integrieren.

Daher, so Schlömilch weiter, wäre es wünschenswert, „wenn Sie mein Compendium der Höheren Analysis recht oft annoncirten". ${ }^{1}$ Die steigende Nachfrage nach mathematischen Publikationen brachte sowohl den Autoren als auch dem Verlagsbuchhandel ökonomischen Vorteil und so sind bei den Verlagen des ausgehenden 19. Jahrhunderts Spezialisierungsprozesse zu beobachten, die die Mathematik ins Zentrum des verlegerischen Interesses rückten. In der Mitte des 19. Jahrhunderts war die - noch zögerlich umge- setzte - Überzeugung, daß mathematisches Wissen in den verschiedensten Disziplinen nötig war, weit verbreitet. Im Jahr 1856 beispielsweise wurde zum 25-jährigen Jubiläum des Polytechnikums in Hannover ein „poesievoller Festgruß" vorgetragen, in dem es u. a. hieß:

Mathematik, du hohe, reine Wahrheit!

Durchdringst der Technik Feld mit lichter Klarheit,

bis tief zum Schacht, den Grubenlicht erhellt. ${ }^{2}$

Die grundsätzliche Bedeutung der Mathematik als Schlüsseltechnologie für die modernen Naturwissenschaften dokumentierten der physikalische Chemiker und spätere Nobelpreisträger Walther Nernst (1864-1941) und der Mathematiker Arthur Schoenflies (1853-1928) im Vorwort ihrer 1895 erschienenen Einführung in die mathematische Behandlung der Naturwissenschaften. Kurzgefasstes Lehrbuch der Differential- und Integralrechnung mit besonderer Berücksichtigung der Chemie:

1 Schlömilch an Vieweg, 27.10. 1889 (Braunschweig, Universitätsbibliothek, Verlagsarchiv Vieweg, V 15: 87, 311 S, Nr. 150).

2 Zit. nach König, Wolfgang: Künstler und Strichezeichner. Konstruktions- und Technikkulturen im deutschen, britischen und französischen Maschinenbau zwischen 1850 und 1930, Frankfurt a. M.: Suhrkamp 1999, S. 54. 
Allgemein kann man sagen, dass eine naturwissenschaftliche Disziplin die Methoden der höheren Mathematik zur Erweiterung und Vertiefung der durch direkte Beobachtungen gewonnenen Ergebnisse um so häufiger zu Rate zieht, je weitere Fortschritte die theoretische Bearbeitung der unmittelbaren Versuchsresultate macht. Im besonderen beginnt gerade die neuere Entwicklung der theoretischen Chemie sich die Methoden der höheren Mathematik nutzbar zu machen. So bemerkt z. B. Herr H. Jahn in der Vorrede zu dem soeben erschienenen Grundriss der Elektrochemie: „Auch die Chemiker müssen sich allmählich an den Gedanken gewöhnen, dass ihnen die theoretische Chemie ohne die Beherrschung der Elemente der höheren Analysis ein Buch mit sieben Siegeln bleiben wird. Ein Differential- oder Integralzeichen muss aufhören, für den Chemiker eine unverständliche Hieroglyphe zu sein, ... [sic] wenn er sich nicht der Gefahr aussetzen will, für die Entwicklung der theoretischen Chemie jedes Verständnis zu verlieren. Denn es ist ja ein fruchtloses Bemühen, in seitenlangen Auseinandersetzungen halb klar machen zu wollen, was eine Gleichung dem Eingeweihten in einer Zeile sagt.“3

Wissenschaftliche Publikationen lassen sich in unterschiedliche Formen mit spezifischen Zielgruppen unterteilen. Für die Mathematik sind diese Formen mit ihren Funktionen noch detailliert zu untersuchen. Dafür ist es zweckmäßig, sich an die Unterscheidung des Soziologen Eliot Freidson zwischen formalem Wissen und Arbeitswissen anzulehnen. Dabei verstehen wir unter formalem Wissen Wissen, das von Spezialisten entwickelt, in höheren Lehranstalten und Universitäten verbreitet und in einer „Disziplin“ organisiert wird, und unter Arbeitswissen Wissen, das Praktiker (Naturwissenschaftler, Ingenieure, Administratoren, etc.) andernorts, d.h. außerhalb der Disziplin Mathematik, ihren Erfordernissen anpassen und einsetzen können. Neben mathematischer Forschungsarbeit im engeren Sinne lag eine zentrale Grundlage für die Erfolge der Mathematik im damaligen Deutschen Reich in der Transformation formalen mathematischen Wissens bzw. mathematischer Kompetenzen in Arbeitswissen. Hier eröffnen sich der Mathematik und ihren Publikationsformen Märkte außerhalb der eigenen Disziplin, deren Erschließung nicht allein ein zentrales Element in den akademischen Legitimierungsstrategien der Mathematiker darstellt, sondern zudem im Interesse der Verlage liegt, die die Mathematik als Schlüsseltechnologie und Nebenfach vermarkten können.

Entscheidend ist dabei natürlich die Frage, wie formales mathematisches Wissen in benutzbares und erlernbares Arbeitswissen umgesetzt werden kann. Daß dieser Aspekt nicht immer zur Zufriedenheit der Techniker, Ingenieure und Naturwissenschaftler ausfiel, bezeugte 1929 die Klage des neuen Rektors der TH Karlsruhe, des Chemikers Alfred Stock (1876-1946), der weitere Reformen im mathematischen Hochschulunterricht wünschte: „Man will die Mathematik weniger als l'art pour l'art, denn als technisches Werkzeug behandelt haben."4

Für Arbeitswissen und formales Wissen sowie alle Wissensformen zwischen diesen Polen stellt sich die gleiche grundsätzliche Frage: welches Wissen wird gebraucht und wie wird es kodifiziert, d. h. insbesondere wie wird es lehrbar, lernbar, verwendbar und verkaufbar gemacht? Die Verlage benötigten mit fortschreitender Entwicklung und Diversifizierung der Mathematik zunehmend Entscheidungshelfer, die mathematische Manuskripte beurteilen, eventuell Themen anregen und den Bedarf an mathematischen Publikationen auf dem Buchmarkt einschätzen konnten. Im Zuge einer stärkeren Spezialisierung in der Verlagsbranche waren alle wissenschaftlichen Verlage mehr und mehr auf Unterstützung angewiesen. Daher begegnet uns ab dem Ende des 19. Jahrhunderts eine Erscheinung, ohne die auch heute kein mathematischer Verlag auskommt: die des Verlagsberaters. Der Typus und die bedeutende Funktion des (mathematischen) Verlagsberaters sowohl für die Disziplin als auch für die Verlage wurden in der historischen Forschung bisher kaum herausgearbeitet oder gewürdigt.

Wie wohl alle Wissenschaftler, äußern sich Mathematiker in Briefen an ihre Verleger häufig und gern zu neuen Plänen und Projekten. Doch nicht jeder Rat macht einen Berater aus; nicht jeder vereinzelten Idee folgt eine Vision, die sich in eine Reihe oder gar ein Programm umsetzen ließe; und - nicht jeder Wissenschaftler kann oder möchte ein Ratgeber sein.

Eine naheliegende Lösung des Problems, Verlagen mathematischen Sachverstand zu verschaffen, ist der Kontakt zu den Mathematikern am Ort: der Verlag Friedrich Vieweg in Braunschweig nutzte in den 1880er und 1890er Jahren u. a. die Kompetenz von Richard Dedekind (1831-1916); der Verlag B. G. Teubner hat sich in den 1890er Jahren in Leipzig immer wieder an Friedrich Engel (1861-1941) gewandt; der Julius Springer Verlag, der in den 1880er und 1890er Jahren ohne Fortune versuchte, in den mathematischen Markt zu kommen, plante 1914, unmittelbar vor Kriegsausbruch, einen neuen Versuch mit

3 Nernst, Walther/Schoenflies, A.: Einführung in die mathematische Behandlung der Naturwissenschaften. Kurzgefasstes Lehrbuch der Differential- und Integralrechnung mit besonderer Berücksichtigung der Chemie, München/Leipzig: Wissenschaftlicher Verlag von Dr. E. Wolff 1895, S. Vf.

4 Stock, Alfred: Die Technische Hochschule am Scheidewege. Rede gehalten anläßlich des Rektoratswechsels am 30. November 1929, Karlsruhe: Druck und Verlag C. F. Müller 1929 [= Karlsruher akademische Reden 5], S. 14. 

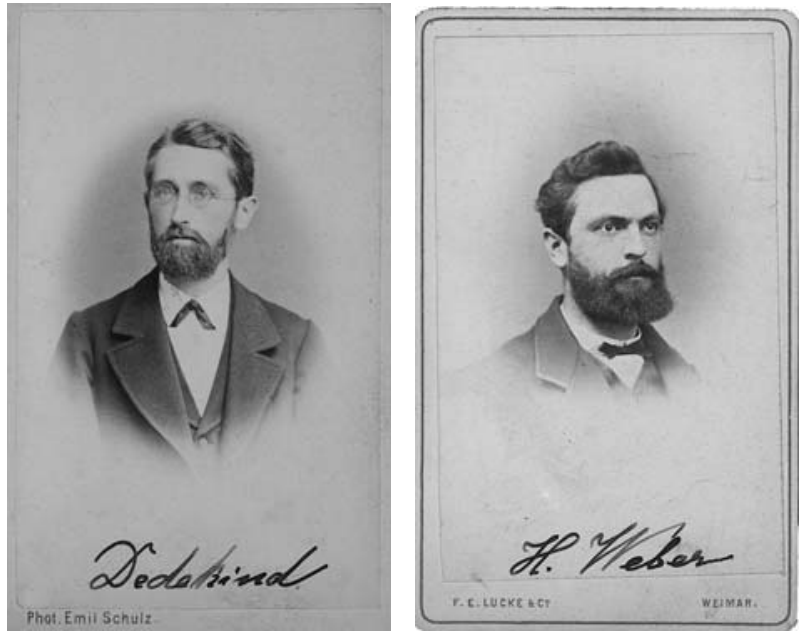

Richard Dedekind (1831-1916)

Heinrich Weber (1842-1913)

dem Berliner Mathematiker Leon Lichtenstein (18781933) als Berater. Nach dem Ersten Weltkrieg ermöglichte die schnelle Verbreitung des Telephons auch die enge, häufige und kurzfristige Abstimmung mit Beratern außerhalb des Verlagsstandortes: Richard Courant (1888-1972) in Göttingen und Ferdinand Springer (1881-1965) in Berlin haben schon ab 1919 häufig miteinander telephoniert.

Aus den professionellen Verbindungen zwischen Beratern und Verlegern erwuchsen nicht selten auch persönliche Beziehungen, manchmal sogar langjährige Freundschaften, allerdings auch konfliktreiche Spannungen, die der nicht immer harmonierenden Interessenlage beider Parteien entsprangen. Honorarfragen, Auflagenhöhen, Werbemaßnahmen und Erscheinungstermine gehören zu den immer wieder kontrovers diskutierten Themen. Die gegenseitige respektvolle Anerkennung der Leistungen auf Autorenwie Verlegerseite kommt jedoch in vielen Korrespondenzen deutlich zum Ausdruck, und die Wertschätzung des verlegerischen Engagements führte auch zu akademischen Ehrungen. Im August 1906 bedankte sich der Verleger und langjährige Schatzmeister der DMV Alfred Ackermann-Teubner (1857-1940) bei Friedrich Engel, der mittlerweile in Greifswald lehrte, für die eben von der dortigen Philosophischen Fakultät erhaltene Ehrendoktorwürde, die insbesondere mit den „Verdiensten“ begründet wurde, „die er

sich als Verlagsbuchhändler um die mathematischen Wissenschaften erworben hat". ${ }^{5}$ Ackermann-Teubner schrieb:

Sie haben mir, sehr verehrter Herr Professor, eine große Freude und Ehre bereitet, die mich durchaus überraschte. In den Zeitungen las ich auch so etwas wie von Weltmeisterschaft. Das ist ja viel zu viel Lob. Ich lebe ja in der Tat ganz in Ihrer schönen Wissenschaft, suche meinen Verlag auf mathematischem Gebiete immer mehr zu vertiefen und weiter auszubauen, aber eine solche Ehrung habe ich wirklich nicht verdient. Sie wird mich immer an die schöne Zeit erinnern, als Sie noch in Leipzig waren, und ich Sie häufig bei B. G. Teubner begrüßen durfte, um Sie nach allem Möglichen und Unmöglichen in mathematicis auszufragen. Auf diese Weise ist ja auch manches schöne Werk des Teubnerschen Verlages zufolge Ihrer freundlichen Anregung zustande gekommen. ${ }^{6}$

Solche persönlichen Begegnungen zwischen Verleger und Berater hinterlassen ebenso wie die Medien Telephon und e-mail weniger Spuren als es den Historikern heute lieb ist.

Im ausgehenden 19. Jahrhundert setzte man bei Vieweg auf Richard Dedekind, der sich die beratende Funktion offenbar mit Robert Fricke (1861-1930) in Braunschweig und Heinrich Weber (1842-1913) in Göttingen und später in Straßburg teilte. Ein angefragtes Gutachten über ein Manuskript konnte Dedekind Ende 1893 einmal nicht liefern: „Ich möchte deshalb empfehlen“, schrieb Dedekind an Vieweg,

in dieser Angelegenheit lieber Herrn Prof. Fricke in Braunschweig oder Herrn Prof. Dr. H. Weber in Göttingen zu Rathe zu ziehen, der ohne Zweifel besser im Stand ist, die gewünschte Auskunft zu ertheilen. ${ }^{7}$

Weber, der wie Dedekind zu den Autoren des Verlags zählte, hat Vieweg des öfteren Ratschläge erteilt, aber dabei wiederholt betont,

dass ich in der Frage durchaus incompetent bin, ob sich irgend ein Werk zu einer buchhändlerischen Speculation eignet, und dass also meine Urtheile sich immer nur auf den wissenschaftlichen Werth des Inhalts beziehen. ${ }^{8}$

Mit der Frage nach den Absatzmöglichkeiten ist ein entscheidender Punkt angesprochen, der im Hause Teubner bis zum Ausbruch des Ersten Weltkrieges zunehmend problematisch wurde: zwar dominierte

5 Engel und Thomé im Antrag an die Philosophische Fakultät vom 23. Juni 1906 (Greifswald, Universitätsarchiv, I-300).

6 Teubner an Engel in Greifswald, 7. August 1906 (Gießen, Universitätsarchiv, Nachlaß Engel, NE 120103).

7 Dedekind an Vieweg, 12. November 1893 (Braunschweig, Universitätsbibliothek, Verlagsarchiv Vieweg, 311 D, Nr. 30 ).

8 Weber an Vieweg, 21. Juli 1901 (Braunschweig, Universitätsbibliothek, Verlagsarchiv Vieweg, V1W: 52).

9 Dazu Schneider, Ute: Mathematik im Verlag B. G. Teubner - Strategien der Programmprofilierung und der Positionierung auf einem Teilmarkt während des Kaiserreichs, erscheint in: dies./Estermann, Monika (Hg.): Wissenschaftsverlage zwischen Professionalisierung und Popularisierung, Wiesbaden: Harrassowitz Verlag (Wolfenbütteler Schriften zur Geschichte des Buchwesens), im Druck.

10 Verhandlungen des 3. Internationalen Mathematiker-Kongresses: Heidelberg, 8.-13. August 1904, hgg. v. Adolf Krazer, Leipzig 1905 , S. 39. 


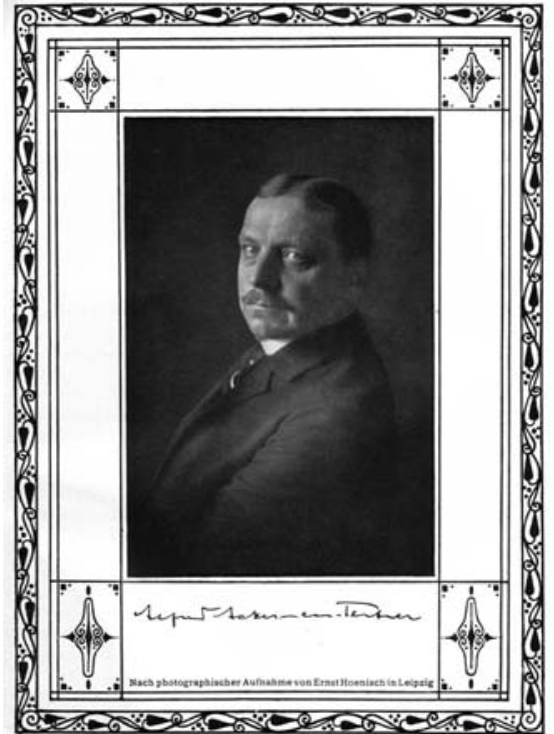

Alfred Ackermann-Teubner (1857-1940)

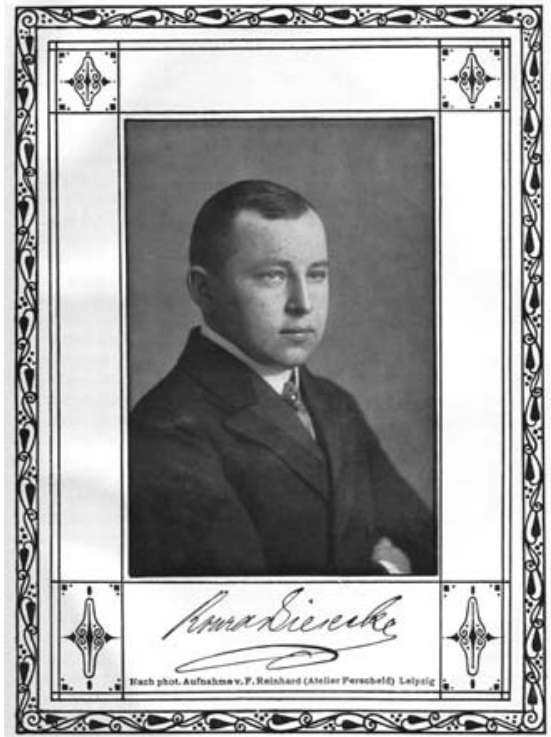

Konrad Giesecke (1878-1931) die Teubnersche Firma um die Jahrhundertwende den mathematischen Markt ${ }^{9}$ und galt als ,stets hilfsbereite Freundin unserer Wissenschaft", ${ }^{10}$ wie Heinrich Weber 1904 zum Abschluß des Internationalen Mathematiker-Kongresses in Heidelberg erklärte, aber nicht jedes wissenschaftlich hochstehende oder verdienstvolle Werk konnte ein verlegerischer Erfolg werden.

Besonders brisant wurde die ökonomische Situation für die Verleger nach dem Ersten Weltkrieg, als sich internationale Sanktionen und die Isolation der Wissenschaft in Deutschland direkt auf den Absatz niederschlugen und durch die Inflation die Produktionszahlen auf dem deutschen wissenschaftlichen Buchmarkt drastisch sanken. Der Buch- und Zeitschriftenvertrieb ins Ausland war erheblich erschwert oder gar nicht mehr möglich. Das Problem der schwindenden Kaufkraft der Studierenden und der Hochschullehrer bei gleichzeitiger Verteuerung der Bücher betraf die gesamte Verlagsbranche. So kamen wissenschaftliche Verlage mehr und mehr in finanzielle Bedrängnis, allein das Schulbuchgeschäft versprach weiterhin relativ kontinuierliche Einnahmen. Eine Folge war die strenge Ausrichtung auf ein Kerngeschäft, die bei fast allen Wissenschaftsverlagen zu beobachten ist. Ackermann-Teubners Nachfolger im mathematischnaturwissenschaftlichen Segment des Hauses Teubner war Konrad Giesecke (1878-1931). Er war offenbar weder in der Lage, diplomatisch mit Autoren umzugehen, noch bereit, sich im gleichen Maß wie sein Vorgänger für die Mathematik zu engagieren. Er klagte Anfang 1920 gegenüber Friedrich Engel, es habe die frühere

Art der Verlagsführung, bei der die wirtschaftlichen Gesichtspunkte vollständig außer acht gelassen, [...] eben dazu geführt, daß die mathematischnaturwissenschaftliche Verlagsabteilung, wie ich Ihnen nunmehr vertraulich mitteilen muß, in den letzten zehn Friedensjahren trotz der guten Einkünfte, die sie auch von verschiedenen Unterrichtswerken gehabt, stets mit zum Teil recht erheblichen Verlusten gearbeitet, sodaß unsere Firma überhaupt nicht hätte weiter bestehen können, wenn die philologisch-pädagogische Abteilung unter der erfolgreichen Leitung meines Bruders nicht für einen entsprechenden Ausgleich gesorgt hätte. ${ }^{11}$

Unabhängig davon, ob Gieseckes ernüchternde Einschätzung zutraf, daß die Mathematik ein unwirtschaftlicher Programmsektor war, würde doch der Idealtypus des Beraters dem Verleger sowohl in Hinsicht auf die wissenschaftliche Qualität als auch auf den zu erwartenden Absatz eines Werkes annähernd verläßliche Auskunft geben können. Daß es dem Springer Verlag gelungen ist, Teubner nach dem Ersten Weltkrieg als führenden mathematischen Verlag abzulösen, liegt nicht allein darin begründet, daß Ackermann-Teubner sich ab 1916 aus dem Geschäft zurückgezogen hatte und Giesecke sich äußerst ungeschickt verhielt, sondern wesentlich darin, daß Ferdinand Springer mit Richard Courant einen Berater gewinnen konnte, der die Rückendeckung von Felix Klein und David Hilbert hatte und darüber hinaus auch wirtschaftlich dachte. Das Haus Springer, das sich in den Jahren nach dem Tod des Verlags-

11 Giesecke an Engel, 2. Februar 1920 (Gießen, Universitätsarchiv, Nachlaß Engel, NE 120244). 


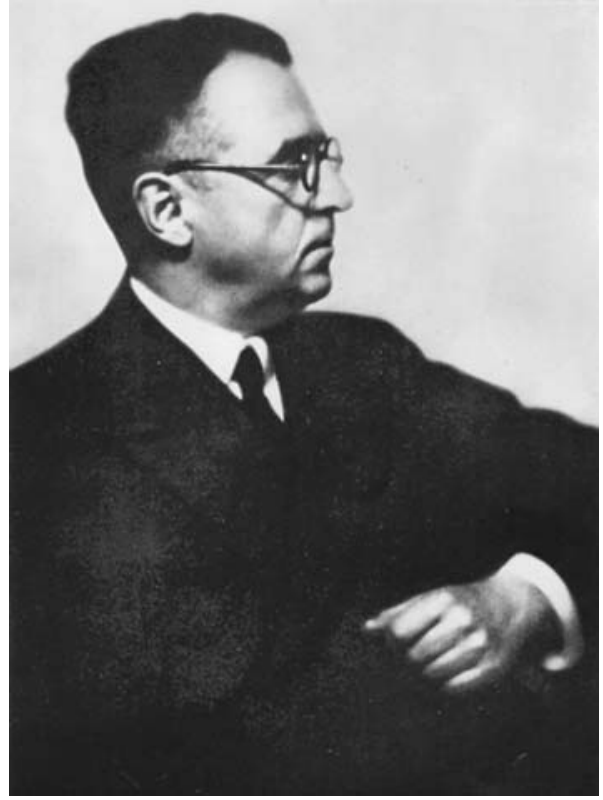

Ferdinand Springer (1881-1965) in den 1930er Jahren

gründers (1877) besonders auf dem Gebiet der technischen Literatur profilierte und geschickte Marktpolitik betrieb, hatte schon im späten 19. Jahrhundert Ambitionen gehabt, auch Mathematik zu verlegen. Springer war mit seinen Bemühungen aber weitgehend erfolglos geblieben, was möglicherweise die Konsequenz eines fehlenden Beraters war. Darauf bezog sich Ferdinand Springer als er im Juni 1914 an den bei Siemens tätigen Berliner Privatdozenten Leon Lichtenstein schrieb, es sei sein „lebhafter Wunsch, allmählich etwas mehr in die Mathematik hineinzukommen, und ich würde es dankbar begrüßen, wenn Sie mich hierin etwas unterstützen könnten". ${ }^{12}$ Lichtenstein entwarf prompt ein ausführliches Publikationsprogramm, das Springer sogleich interessiert aufnahm. Doch der Kriegsausbruch vereitelte vorerst ein zielgerichtetes gemeinsames Vorgehen. Unter Federführung Lichtensteins wurde allerdings noch 1917 bei Springer die Mathematische Zeitschrift in bewußter Konkurrenz zu Teubners traditionsreichen Mathematischen Annalen gegründet. Mit dieser Zeitschriftengründung signalisierte der Verlag sein ernsthaftes Interesse an der Mathematik und konnte leichter Autorenkontakte auf- und ausbauen. Zunächst war die Investition in eine neue, von der Fachwelt noch nicht anerkannte Zeitschrift ein hohes verlegerisches Risiko, zumal in Kriegszeiten, aber diese Strategie erwies sich als richtiger Schritt bei der Profilierung eines neuen Programmschwerpunktes, der mit Unterstützung
Lichtensteins verwirklicht werden sollte. Im Mai 1918 bestätigte Springer Lichtenstein schriftlich

unsere bei Ihrem letzten Besuch mündlich getroffene Vereinbarung, dass Ihnen für Ihre beratende Tätigkeit im Interesse meiner Firma ein jährlicher Betrag von M. 1500.--, zahlbar in Halbjahresraten, zur Verfügung gestellt werden soll, und zwar beginnend vom 1 . Oktober $1918 .^{13}$

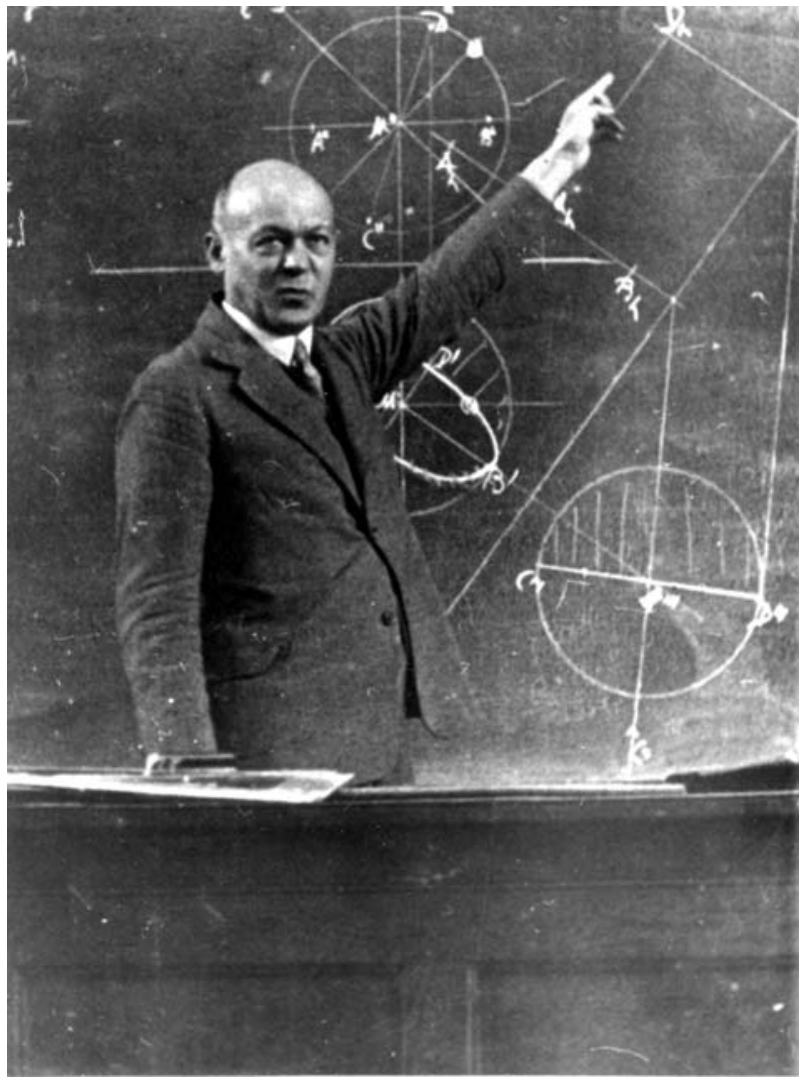

Leon Lichtenstein (1878-1933) in den 1930er Jahren

Spätestens zu diesem Zeitpunkt ist der Typus des bezahlten mathematischen Verlagsberaters etabliert. Diskutiert wurde er schon Ende des 19. Jahrhunderts, als Ackermann-Teubner für seinen Verlag Arnold Sommerfeld und Walther Dyck als Berater gewinnen wollte. Den Vorschlag ,einer Honorirung der einzelnen Bogen der von uns veranlaßten Werke" hielt Dyck, wie er Sommerfeld Ende 1898 unumwunden mitteilte, für den falschen Weg. „Ich faße“, so Dyck weiter,

die ganze Aufgabe, die wir uns hier gestalten müßen, allgemeiner auf - der Rat betr. eines speziellen Buches und dessen Verwirklichung durch einen von uns vorgeschlagenen Autor ist dabei ein zu spezielles Moment.

12 Springer an Lichtenstein, 15. Juni 1914 (Heidelberg, Verlagsarchiv Springer, Abteilung B [1912-1936], M 69).

13 Springer an Lichtenstein, 20. Mai 1918 (Heidelberg, Verlagsarchiv Springer, Abteilung B [1912-1936], M 69). 1500 Mark entsprachen ungefähr 4 Monatsgehältern eines gelernten Arbeiters. 


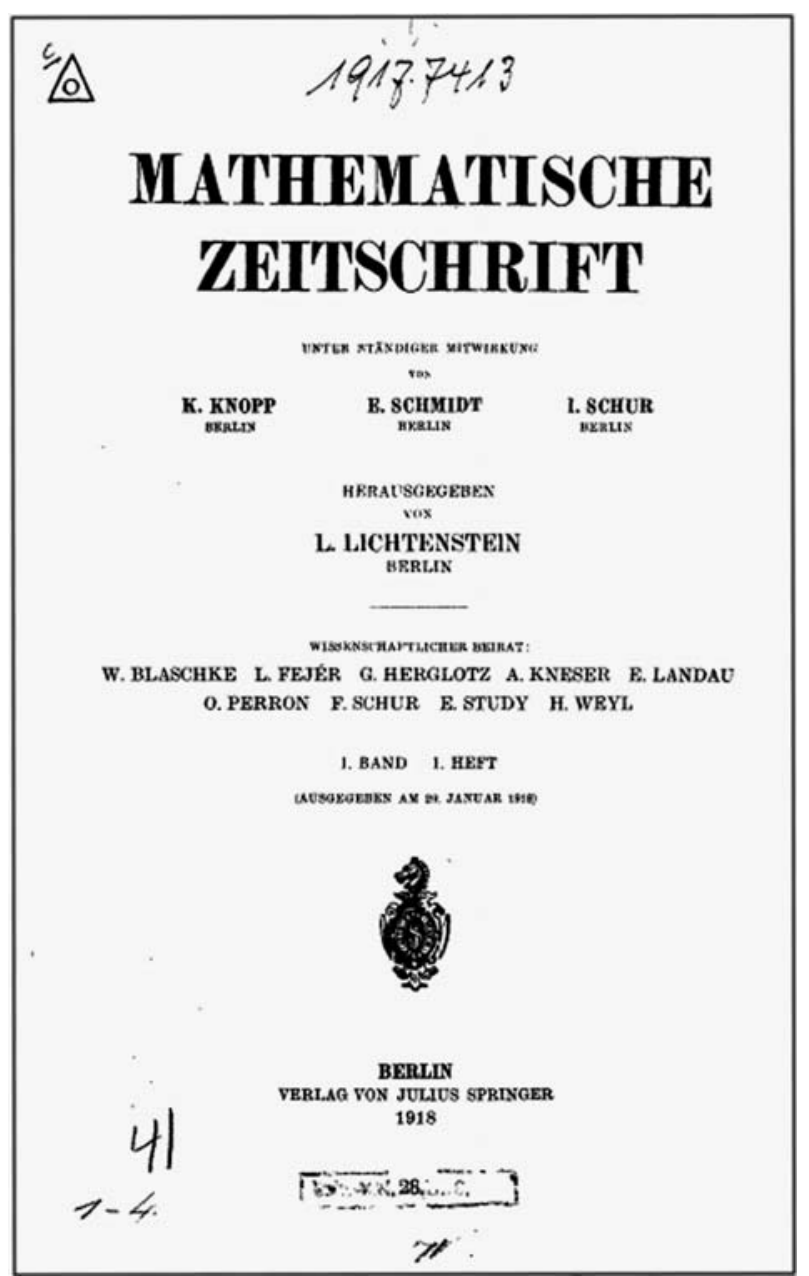

Titelblatt des ersten Heftes der Mathematischen Zeitschrift vom Januar 1918

Unsere Tätigkeit ist zuvörderst eine allgemein orientirende, dann aber eine negative abratende, ebenso wie eine positive zuratende.

Dyck erklärte sich „fürs erste“ mit der Honorierung je Bogen einverstanden, aber „für später [. . .] würde mir eine generelle Honorierung richtiger erscheinen."14

Auch Springers Expansionsbemühungen wurden nicht nur durch einen einzigen Berater unterstützt. Zur gleichen Zeit als Springer seine Vereinbarung mit Lichtenstein traf, hatte er mit Richard Courant bereits einen weiteren Berater gewonnen. Mit ihm plante er eine neue Reihe, die „Grundlehren der mathematischen Wissenschaften in Einzeldarstellungen mit besonderer Berücksichtigung der Anwendungsgebiete". Die ab 1921 erscheinenden Grundlehren sind als gelbe Reihe oder - wegen ihrer Unausweichlichkeit -

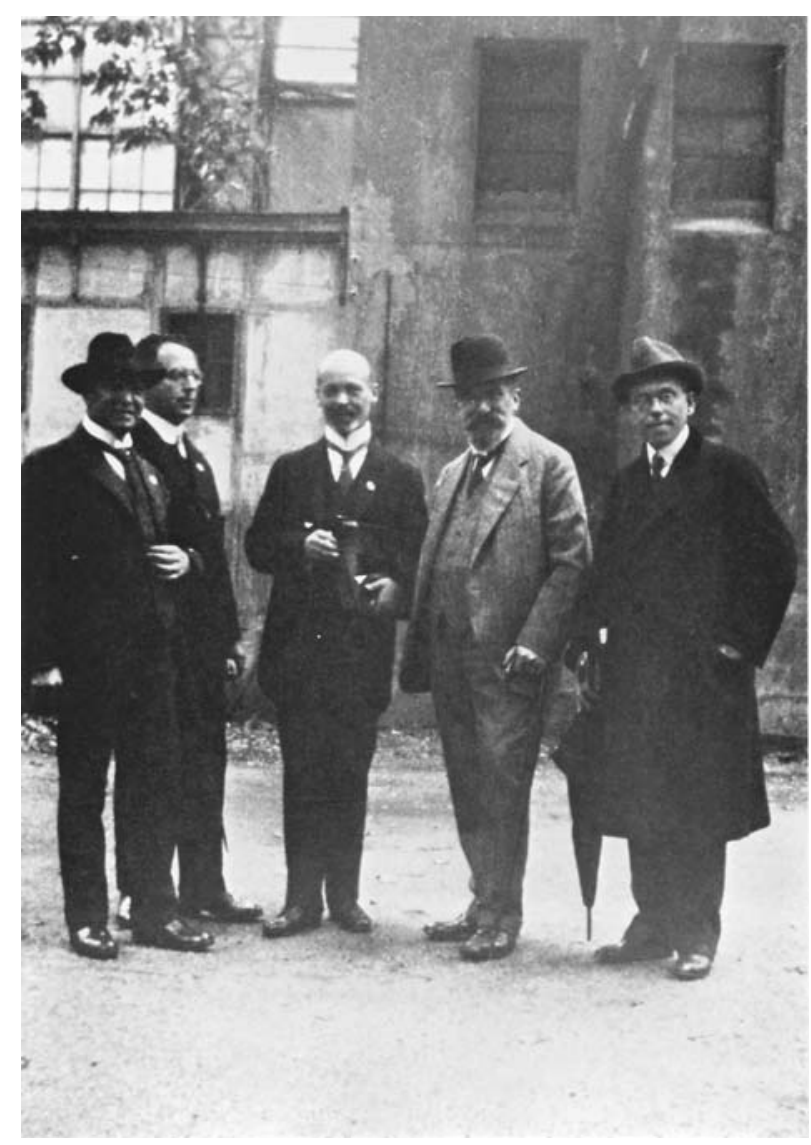

DMV-Tagung 1920 oder 1921: Ludwig Schlesinger, Ferdinand Springer, Leon Lichtenstein, Kurt Hensel und Leopold Fejer (von links nach rechts)

auch als „gelbe Gefahr" bekannt geworden und wurden von Courant gemeinsam mit Carl Runge, Wilhelm Blaschke und Max Born herausgegeben. Wesentlich für den Erfolg war die Protektion durch Hilbert, den Courant als Autor der Reihe gewinnen konnte und der dem Projekt volle Rückendeckung gab. Entsprechend enthusiastisch schrieb Courant im Juli 1918 an Springer: „In jedem Falle ist die Gewinnung von Hilbert ein Riesenfortschritt.“15

Kennzeichnend für den Führungswechsel im mathematischen Publizieren von Teubner zu Springer, der Stoff für eine eigene Untersuchung bietet, sind drei weithin sichtbare Projekte:

1. Der Übergang der Mathematischen Annalen von Teubner zu Springer 1919/20, den Ackermann-Teub-

14 Dyck an Sommerfeld, 29. Dezember 1898, in: Eckert, Michael/Märker, Karl (Hg.): Arnold Sommerfeld: Wissenschaftlicher Briefwechsel, Bd. 1: 1892-1918, Berlin/Diepholz/München 2000, S. 102-104.

15 Courant an Springer, 2. Juli 1918 (Heidelberg, Verlagsarchiv Springer, Abteilung B [1912-1936], C 67 I). 
ner in einem Brief an Engel im Januar 1920 so kommentierte:

Der Verlust der Annalen ist ein überaus beklagenswerter, der Firma Springer ist ein Geschenk des Himmels in den Schoss gefallen, sie ist über Nacht in der Tat $\mathrm{zu}$ einer Weltfirma geworden. ${ }^{16}$

2. Der Übergang der noch nicht mit allen Bänden vorliegenden Göttinger Gauß-Werke von Teubner zu Springer nach dem Krieg, von Giesecke 1919 mit den unglücklichen Worten begleitet, daß

einzelne Bände der Gauss'schen Werke [...] nach jetzigem Vorrat und dem Absatz der letzten Jahre noch mehrere Jahrhunderte ohne Aussicht auf Verwendung lagern würden.

Daher sei zu überlegen, „ob die über den Durchschnitt des Vorrates der anderen Bände hinaus lagernde Anzahl makuliert werden könnte. "17 Das durfte der Verleger zwar denken, aber kaum den Göttinger Mathematikern schreiben.

3. Die Publikation der Gesammelten Abhandlungen von Felix Klein in den Jahren 1921 bis 1923, die nach Einschätzung der Herausgeber bewies, daß die Firma Springer

trotz aller sich häufenden Hemmungen bemüht ist, das Ansehen und die Geltung der deutschen Wissenschaft zu erhalten und zu fördern. Sie kann des Dankes aller Mathematiker gewiß sein. ${ }^{18}$

Mit diesen drei Projekten konnte der Springer Verlag seine Reputation enorm steigern, aber sein rascher Erfolg rief alsbald auch deutliche Kritik und Befürchtungen hervor. Springers Führungsrolle war nicht unumstritten, weil ein Monopol seines Verlags befürchtet wurde. Tatsächlich geriet der Springer Verlag in der Weimarer Zeit zunehmend in die öffentliche Kritik, besonders auch im Ausland. Am 23. Juli 1928 hatte die schwedische Zeitung Svenska Dagbladet geschrieben:

Der außerordentlich produktive Springersche Verlag überschwemmt die wissenschaftliche Welt auf allen Forschungsgebieten. Der Inhalt ist oft von hoher Klasse, die Ausstattung von noch höherer, aber am allerhöchsten in der Reihe steht der Preis, der nicht selten phantastisch erscheint. ${ }^{19}$

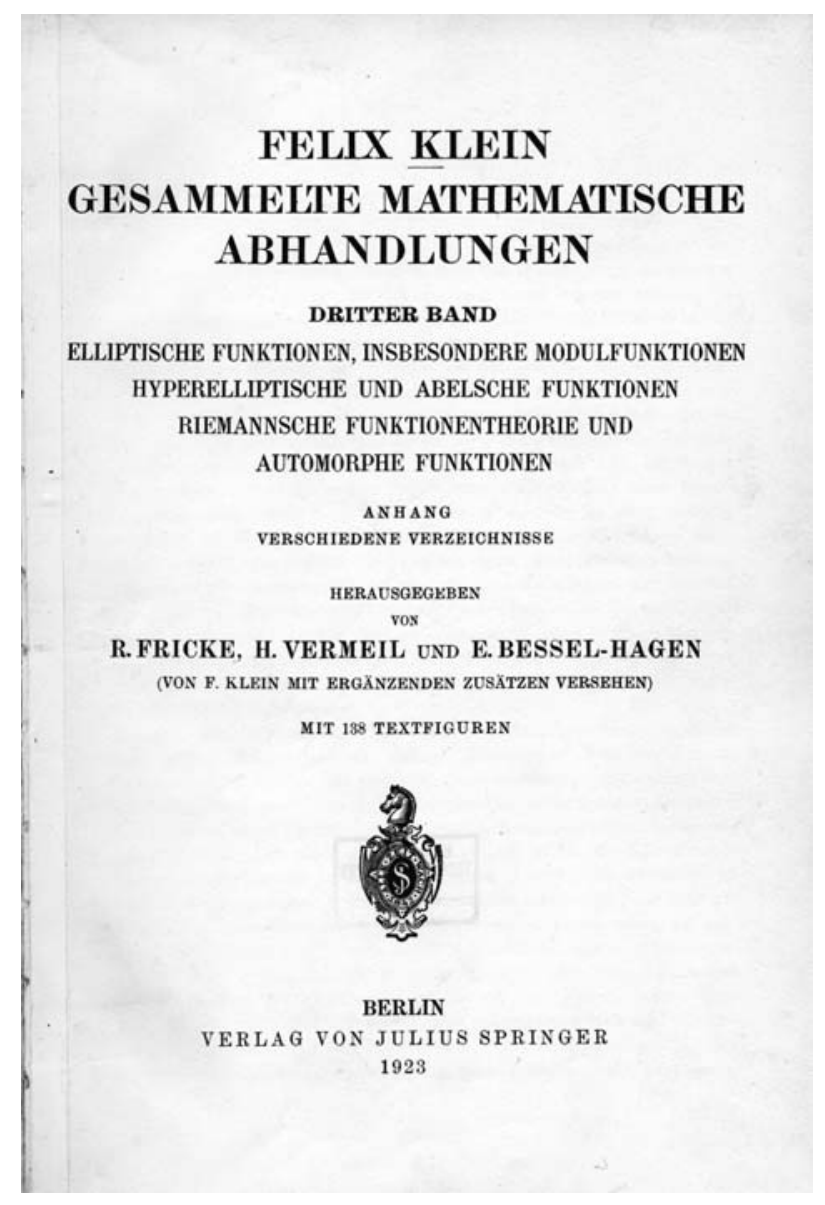

Titelblatt von Felix Klein: Gesammelte Abhandlungen, 1923

Von Seiten der Mathematik wurde schon 1921 der Vorwurf erhoben, Springer strebe „ein Monopol auf dem Gebiete der Mathematik" an, um schließlich „die Autoren bezw. die Abnehmer der mathematischen Literatur auszubeuten". ${ }^{20}$ Ferdinand Springer hat diese Anschuldigung entschieden zurückgewiesen und unter anderem entgegnet, es sei „doch klar, dass ein Verleger, der auf wirtschaftliche Ausbeutung ausgeht, sich nicht gerade die Mathematik als Gebiet seiner Tätigkeit aussuchen" werde. ${ }^{21}$ Dennoch mag es Balsam für seine Seele gewesen sein, als Courant ihm im Februar 1921 schrieb, Hilbert lasse ausdrücklich „erklären, dass er aufs entschiedenste jedes auch noch

16 Ackermann an Engel, 30. Januar 1920 (Gießen, Universitätsarchiv, Nachlaß Engel, NE 120245).

17 Giesecke an Klein, 4. April 1919 (Gießen, Universitätsarchiv, Nachlaß Engel, NE 120235A).

18 Klein, Felix: Gesammelte mathematische Abhandlungen, 3 Bände, Berlin: Verlag von Julius Springer 1921-1923, Band II, hgg. v. Fricke und Vermeil, 1922, S. IV.

19 Vgl. die deutsche Übersetzung des Artikels in: Springer, Ferdinand: Die Preise der deutschen wissenschaftlichen Zeitschriften und das Ausland. Nach einem am 9. November 1928 vor der Arbeitsgemeinschaft wissenschaftlicher Verleger gehaltenen Referat. Mit einem Anhang: Beiträge zur Psychologie des In- und Auslandes. Als Manuskript gedruckt 1928, Anlage 1, S. 25.

20 Springer an Krazer, 9. Februar 1921, z. K. Klein (Göttingen, Staats- und Universitätsbibliothek, Nachlaß Felix Klein, Nr. 81-100).

21 Ebd. 


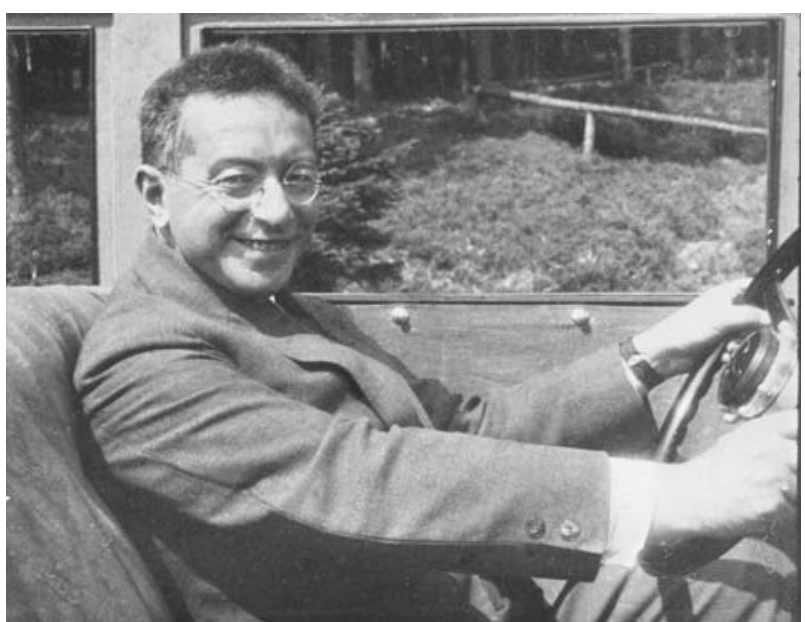

Richard Courant (1888-1972) in seinem ersten Auto 1929: „Ich habe endlich heute meine Autoprüfung bestanden und kann jetzt ohne Aufsicht auf die Menschheit losgelassen werden." (Mai 1929)

so leichte Misstrauen gegen Ihren Verlag ablehnt und völlig hinter Ihnen steht. ${ }^{22}$ Die Solidarität galt einem Verleger, der in wirtschaftlicher Krise das mathematische Publikationswesen zunächst ohne Rücksicht auf einen zu erwartenden Profit unterstützte und damit einen Vertrauensvorschuß bei den Mathematikern genoß, den er später möglicherweise in ökonomischen Gewinn umwandeln konnte.

Inmitten dieser Entwicklungen stand Courant in dauernder enger Fühlung mit Springer, dem er im Juni 1921 versicherte:

Wenn ich Ihnen mit meinem Rate gelegentlich nutzen könnte, so würde mich dies sehr freuen; irgendwelche Bedenken, dass eine solche Inanspruchnahme eine zu grosse Belästigung darstellen könnte, brauchen Sie wirklich nicht zu haben. Ich weiss sehr wohl, was unsere Wissenschaft an Ihrem Verlage hat, und daraus folgt ohne weiteres die Verpflichtung, Ihnen nach Kräften behilflich zu sein. ${ }^{23}$

Voraussetzung für wissenschaftlichen wie auch ökonomischen Erfolg mußte die inhaltliche Qualitätssicherung sein. So beschrieb Courant nur wenige Tage später sein Rollenverständnis in Abgrenzung vom Teubnerschen Usus:

Teubner hat, als es ihm gut ging, ziemlich kritiklos alles genommen, was ihm angeboten wurde, nicht nur Sachen, die kein Geschäft bedeuten konnten, sondern auch solche, die obendrein wenig Wert für die Wissenschaft besitzen. Ich glaube, Sie recht zu verstehen, wenn ich bei Ihren Anfragen annehme, dass diese beiden Gesichtspunkte Sie interessieren. ${ }^{24}$

Springer trug noch im gleichen Jahr der Tatsache Rechnung, daß Courant mit seinen Göttinger Verbindungen sich zu seinem „Hauptberater in mathematischen und physikalischen Dingen" entwickelt hatte. Die Programmprofilierung war das Ergebnis einer intensiven Zusammenarbeit. „Der Umfang,“ so Springer,

in welchem ich Sie in Anspruch zu nehmen mir erlaube, wird immer grösser, und so kann ich es nicht mehr verantworten, ohne wesentliche Gegenleistung so viel von Ihrer Zeit in Anspruch zu nehmen. Ich möchte Ihnen daher den Vorschlag machen, dass ich Ihnen für die Tatsache, dass Sie mir jederzeit zur Verfügung stehen und dass Sie auch von sich aus mich auf in Ihrem Bereiche bestehende literarische Pläne aufmerksam machen, mir auch sonst Anregungen geben, Ihnen einen festen jährlichen Unkostenersatz zur Verfügung stelle. Ich schlage vor, ihn zunächst auf M. 1500.- für das Quartal zu bemessen, und zwar vom 1. Oktober 1921 an gerechnet. $^{25}$

Damit waren zugleich die Erwartungen des Verlegers wie auch die Aufgaben eines Beraters klar umrissen, und Courant, der sie mit Leidenschaft wahrnahm, akzeptierte das Angebot gern, zumal die in Aussicht genommene jährliche Entschädigung zu diesem Zeitpunkt dem Jahresgehalt eines gelernten Arbeiters entsprach. ${ }^{26}$

Eine genaue Analyse seiner verzweigten Tätigkeit für Springer, auch im Hinblick auf die Wirtschaftlichkeit der von ihm angeregten Projekte, steht noch aus. Hilberts Beistand blieb dabei wesentlich. Während die Publikation von Kleins Gesammelten $A b$ handlungen durch Springer noch als Schachzug gedeutet werden konnte, den er auch ohne Aussichten auf pekuniären Erfolg durchführen mußte, wenn er im mathematischen Markt reüssieren wollte, so zeigt die Herausgabe der Hilbertschen Gesammelten Abhandlungen zehn Jahre später einen selbstbewußten Verlag, der sich bereits die bleibende Anerkennung der Mathematiker in Deutschland erworben hatte. Diese Anerkennung fand Ausdruck in der Verleihung der Ehrendoktorwürde der Göttinger mathematisch-naturwissenschaftlichen Fakultät an Ferdinand Springer im Januar 1930. In ihrem gemeinsamen Glückwunschbrief betonten Hilbert, Cou-

22 Courant an Springer, 16. Februar 1921 (Heidelberg, Verlagsarchiv Springer, Abteilung B [1912-1936], C 67 I).

23 Courant an Springer, 18. Juni 1921 (Heidelberg, Verlagsarchiv Springer, Abteilung B [1912-1936], C 67 I).

24 Courant an Springer, 24. Juni 1921 (Heidelberg, Verlagsarchiv Springer, Abteilung B [1912-1936], C 67 I).

25 Springer an Courant, 21. Oktober 1921 (Heidelberg, Verlagsarchiv Springer, Abteilung B [1912-1936], C 67 I).

26 Dazu s. Zahlen zur Geldentwertung in Deutschland 1914 bis 1923. Bearbeitet im Statistischen Reichsamt, Berlin: Verlag von Reimar Hobbing 1925, S. 40. 


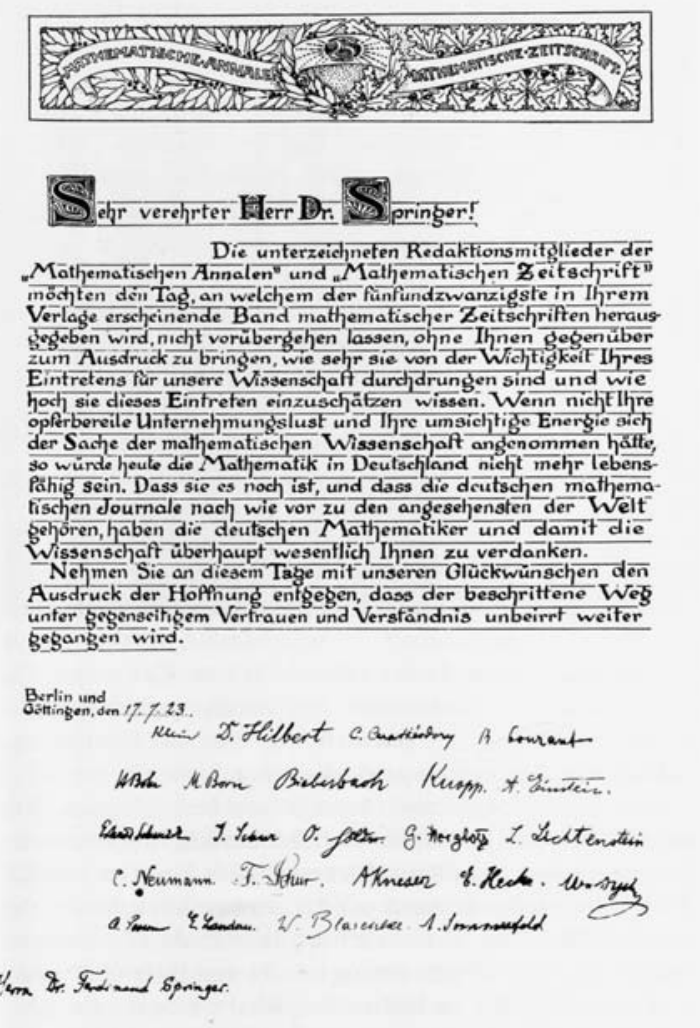

Glückwunschadresse zur 25. Nummer der Mathematischen Zeitschrift und der Mathematischen Annalen im Springer Verlag, Juli 1923

rant, Landau und Herglotz die Funktion des Verlegers im wissenschaftlichen Kommunikationsprozeß:

Sie wissen ja seit langem, wie hoch wir Göttinger Mathematiker Ihre Tätigkeit für die mathematische Wissenschaft einschätzen. Hätten Sie nicht nach dem Kriege in Ihrer grosszügigen und weit ausblickenden Art sich in die Bresche gestellt, so wäre der mathematischen Literatur in Deutschland durch das Versagen anderer Verleger ein tödlicher Schlag versetzt worden, der auch auf die Wissenschaft als solche unheilvolle Auswirkungen hätte haben müssen. ${ }^{27}$

Hilbert ließ es sich nach dem Erscheinen des zweiten Bandes seiner Gesammelten Abhandlungen im August 1933 nicht nehmen, Springer seinen Dank mit den Worten auszusprechen:

Die Drucklegung ist wieder so sorgfältig erfolgt und die Ausstattung so prachtvoll ausgefallen wie beim ersten Bande; ich bin wirklich glücklich zu preisen, einen solchen Verleger-Freund zu besitzen. ${ }^{28}$

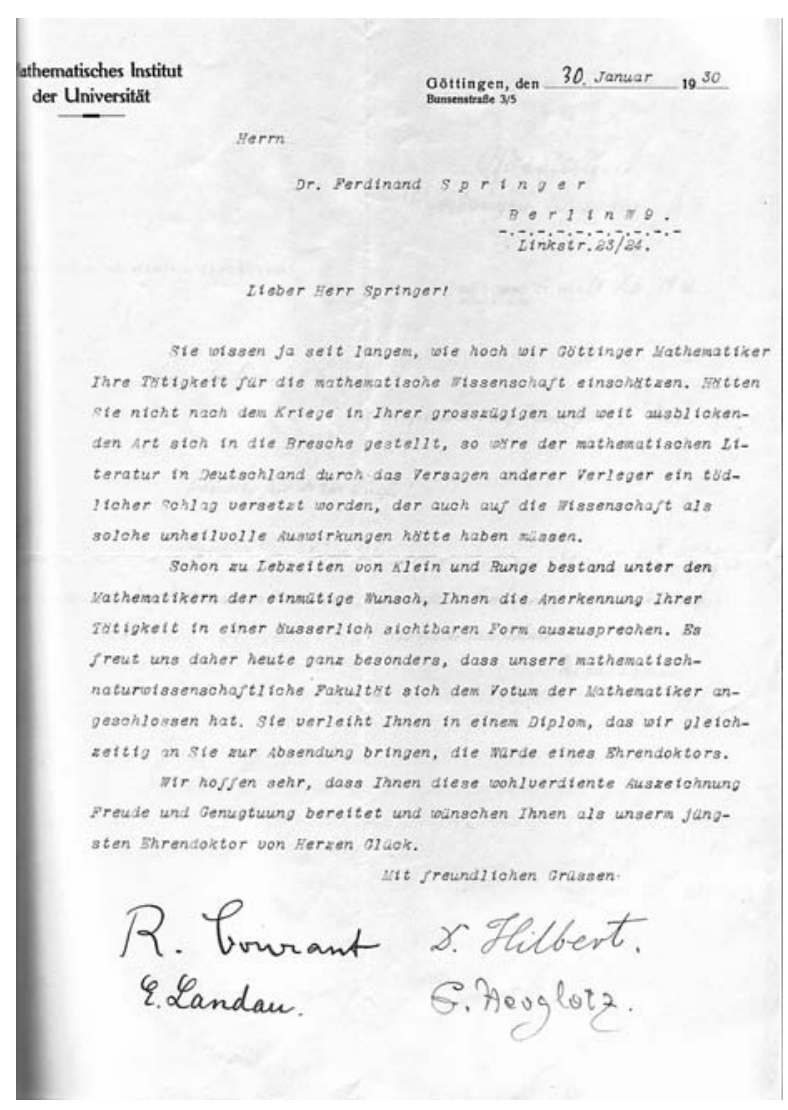

Hilbert, Courant, Landau und Herglotz gratulieren Ferdinand Springer zum Göttinger Ehrendoktor, Januar 1930

Dieser freundliche Ton sollte jedoch nicht darüber hinwegtäuschen, daß im Verhältnis zwischen Mathematikern und ihren Verlegern immer wieder Spannungen auftraten und -treten. Aber vielleicht kann der Blick auf die wechselvolle Geschichte des mathematischen Publizierens dazu beitragen, solche Spannungen nicht überproportional $\mathrm{zu}$ bewerten, auch wenn wohl die Zeiten vorbei sind, in denen ein Ackermann-Teubner erklärte:

Mir stand mein mathematischer Verlag immer wie eine wunderherrliche Statue vor Augen, nicht von mir geschaffen, wohl aber von mir treulichst gepflegt und gehegt. “29

\section{Bildnachweise}

Seite 198: Photoalbum der Hamburger Mathematischen Gesellschaft, SP Geschichte der Naturwissenschaften, Mathematik und Technik, Universität Hamburg / Seite 199: B. G. Teubner 1811-1911. Geschichte der Firma in

27 Hilbert, Courant, Landau und Herglotz an Springer, 30. Januar 1930 (Heidelberg, Verlagsarchiv Springer, FS 31.2). 28 Hilbert an Springer, 2. August 1933 (Heidelberg, Verlagsarchiv Springer, Abteilung B [1912-1936], H 241).

29 Ackermann an Engel, 14. Januar 1920 (Gießen, Universitätsarchiv, Nachlaß Engel, NE 120243). 
deren Auftrag herausgegeben von Friedrich Schulze, Leipzig 1911 / Seiten 200, 201, 203, 204: Archiv des Springer Verlags, Heidelberg / Seite 200: Universitätsarchiv Leipzig: Signatur df 1062

\section{Adresse der Autoren}

Dr. habil. Volker R. Remmert

AG Geschichte der Mathematik und der

Naturwissenschaften

Institut fï Mathematik

FB 08 - Physik, Mathematik und Informatik

Johannes Gutenberg-Universität Mainz

55099 Mainz

remmert@mathematik.uni-mainz.de

PD Dr. Ute Schneider

Institut für Buchwissenschaft

FB 05 - Philosophie und Philologie

Johannes Gutenberg-Universität Mainz

55099 Mainz

uschneid@mail.uni-mainz.de
Volker Remmert unterrichtet Geschichte der Mathematik und der Naturwissenschaften. Seine Forschungsinteressen liegen neben der Geschichte der Mathematik in Deutschland im 19. und 20. Jahrhundert vor allem im Bereich der europäischen Wissenschaftsgeschichte des 16.-18. Jahrhunderts. Dazu ist vor kurzem seine Studie Widmung, Welterklärung und Wissenschaftslegitimierung: Titelbilder und ihre Funktionen in der Wissenschaftlichen Revolution erschienen (Wiesbaden 2005).

Ute Schneider lehrt und forscht zur Verlagsorganisation im 20./21. Jahrhundert, zur Geschichte des wissenschaftlichen Verlags im 19. und 20. Jahrhundert sowie zur Lesergeschichte insbesondere in der Medienkonkurrenz der Weimarer Republik. Zuletzt ist erschienen: Der unsichtbare Zweite. Die Berufsgeschichte des Lektors im literarischen Verlag (Göttingen 2005).
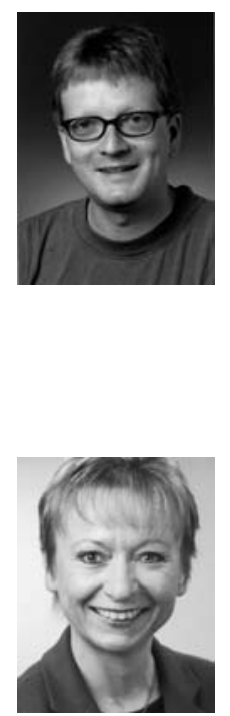

\section{In Mathe war ich immer schlecht ...}

„Auch wenn über dem Eingang der platonischen Akademie in Athen der Sinnspruch ,Niemand, der nicht mathematisch gebildet ist, soll hier eintreten' stand, so ist diese Einsicht in der heutigen akademischen Welt längst nicht mehr selbstverständlich. Auf mich jedenfalls trifft das zu." (in der Laudatio auf Gyburg Radke)
„Mathematiker sind immer schwierige Fälle bei solchen Laudationes" (nach Verlesung der Laudatio auf Felix Otto)

DFG-Präsident Prof. Ernst-Ludwig Winnacker bei der Verleihung der Leibniz-Preise, Berlin, 8. Februar 2006 